\title{
Uso de un sustrato alternativo a la turba para la producción viverística de plantas hortícolas y aromáticas
}

Recibido: 9 outubro 2012 / Aceptado: 19 novembro 2012

(C) IBADER- Universidade de Santiago de Compostela 2012
Resumen Se obtuvieron dos compost a partir de matorral compuesto mayoritariamente por tojo y especies herbáceas, añadiendo en uno de ellos un $5 \% \mathrm{v} / \mathrm{v}$ de gallinaza procedente de una explotación ecológica de puesta. Los compost obtenidos fueron tamizados a $6 \mathrm{~mm}$ y caracterizados física, química y biológicamente teniendo en cuenta los requerimientos para la concesión de la etiqueta ecológica comunitaria para sustratos. Se llevaron a cabo tres ensayos de producción diferentes; con lechuga (Lactuca sativa L.), albahaca (Ocimum basilicum L.) y perejil (Petroselinum crispum (Mill) Fuss) para su comercialización en maceta. Se evaluaron tanto los dos compost elaborados como sus mezclas en proporciones $25 / 75,50 / 50$ y $75 / 25 \%$ $(\mathrm{v} / \mathrm{v})$, con el fin de obtener así distintas proporciones de gallinaza en el sustrato final. De los resultados se desprende que ambos compost cumplen los requisitos para la obtención de la etiqueta ecológica, y que las mezclas son aptas para su empleo en producción de planta hortícola y aromática. En general para los tres cultivos las mezclas que mejor respondieron son aquellas que contaban con $50 \%$ o más de compost con gallinaza en la mezcla.

Palabras clave tojo, Ulex europaeus, gallinaza, etiqueta ecológica, medio de cultivo.

Abstract Two composts were produced from gorse and herbs, and adding to one of them $5 \%(\mathrm{v} / \mathrm{v})$ of poultry manure from an organic egg production farm. The composts were sieved to $6 \mathrm{~mm}$ and characterized physically, chemically and biologically considering the requirements for the award of

Noelia López López · Adolfo López Fabal Universidade de Santiago de Compostela Escola Politécnica Superior

Campus Universitario, 27002-Lugo, España.

E-mail: noelia.lopez.lopez@usc.es the EU Ecolabel for substrates. Three different production trials were conducted with lettuce (Lactuca sativa L.), basil (Ocimum basilicum L.) and parsley (Petroselinum crispum (Mill.) Fuss) for marketing in pots. Mixtures (0/100, 25/75, $50 / 50,75 / 25$ and $100 / 0 \%(v / v))$ were prepared from these two composts to obtain different ratios of poultry manure in the final substrate. The results show that all compost mixtures achieved the requirements to obtain the Ecolabel and that the mixtures were suitable for use in horticultural and aromatic plant production. Overall for the three crops, the best results were obtained with mixtures containing at least $50 \%$ of compost with poultry manure.

Key Words gorse, Ulex europaeus, poultry manure, Ecolabel, growing media.

\section{Introducción}

La turba es el componente más utilizado en la formulación de medios de cultivo. Solamente en el año 2009 en España se consumieron $224.623,8 \mathrm{t}$ de turba, de las cuales $169.537,5$ t fueron importadas, en su mayor parte de Alemania, pero también de Estonia, Letonia, Países Bajos y Lituania (Marchán et al., 2010). Actualmente existe una tendencia creciente a la búsqueda de materiales que puedan sustituirla, por el alto coste de la turba de calidad, y sobre todo por la actual consideración medioambiental de las turberas a nivel europeo. La Directiva 92/43/CEE del Consejo de 21 de mayo de 1992, relativa a la conservación de los hábitats naturales y de la fauna y flora silvestres, protege los ecosistemas de turberas dada su consideración ambiental y la valoración de los mismos como reserva de $\mathrm{CO}_{2}$. Ello ha llevado a que el suministro de turba en Europa se esté desplazando desde Alemania hacia los países del Báltico, empeorando la calidad de la materia prima y aumentando los costes debido a la distancia de transporte.

En este contexto va en aumento el uso como sustrato de compost de diferentes orígenes y características (Abad, 2002; Urrestarazu et al., 2006); uso que implica un alto nivel de exigencias en cuanto a la calidad del compost (Carmona 
\& Abad, 2008). La utilización de productos orgánicos como el compost para la elaboración de sustratos en sustitución de materiales no renovables, permite tanto el uso sostenible de los recursos naturales como la generación del mínimo impacto ambiental. Son muchos los trabajos que contemplan el uso del compost como sustrato, a partir del aprovechamiento de numerosos residuos como los hortícolas (Mendoza-Hernández et al., 2011), restos de poda en combinación con biosólidos (Tapia et al., 2009), lodos residuales (Ortega \& Ordovás, 2011), residuos vitivinícolas (Agulló et al., 2009, Carmona et al., 2009), o incluso a partir de restos de pescado y algas de arribazón (Illera-Vives et al., 2011).

El tojo (Ulex europaeus L.) es un matorral muy abundante en el ambiente húmedo $\mathrm{y}$ de suelos ácidos del $\mathrm{NO}$ de España. Como leguminosa es rica en nutrientes y presenta una relación $\mathrm{C} / \mathrm{N}$ moderada, lo que facilita su compostaje. Algunos trabajos ya han permitido tener constancia de la calidad del compost de tojo (Gómez et al., 2009), así como la posibilidad de su uso como componente de sustrato sustituyendo parcialmente a la turba e incluso como material único (Iglesias et al., 2008; López-López et al., 2011). La incorporación de estiércol de gallinaza al proceso de compostaje incrementa considerablemente el nivel de nutrientes de los compost resultantes, debido a su alto poder fertilizante, al tiempo que permite la valorización de un recurso y la minimización del impacto ambiental que supone su acumulación.

Los objetivos del presente trabajo se concretan en la caracterización de compost a base de tojo (C (compost sin gallinaza) y CG (compost con gallinaza)) y en su evaluación para su uso como sustrato en diferentes cultivos aromáticos y hortícolas ecológicos.

\section{Material y métodos}

Los materiales evaluados se obtuvieron por un compostaje de 9 meses de duración de material de desbroce de matorral de monte, principalmente tojo (Ulex europeaus (L.)) de 3-4 años de edad, recogido con una segadora de mayales. Se obtuvieron 2 tipos de compost diferentes con 2 repeticiones cada uno. El factor diferenciador fue la adición o no de gallinaza ecológica en proporción $5 \%$ (v/v) (C y CG respectivamente).

De los compost obtenidos, se llevó a cabo la evaluación de algunas características físicas, químicas y biológicas de cara a su uso como sustratos, teniendo en cuenta además, los requerimientos de la normativa europea para la obtención de la etiqueta ecológica (Reglamento 2007/64/CE). Se determinó la curva de retención de humedad de los sustratos sometiendo muestras de los mismos a tensiones crecientes $(1,3,5,7,5$ y $10 \mathrm{kPa})$ en una caja de arena (De Boodt et al., 1974). La porosidad total se determinó a partir de la densidad aparente y de la densidad de partícula (UNE-EN-13041:1999).

De los dos compost iniciales (C y CG), se midió la CE en extracto 1:5 (v/v) conforme el método UNE-EN-13652:2002, sobre el extracto de saturación se determinaron el pH, CE,
$\mathrm{P}, \mathrm{NO}_{3}{ }^{-}, \mathrm{NH}_{4}{ }^{+}, \mathrm{Ca}, \mathrm{Mg}, \mathrm{K}, \mathrm{Cu}, \mathrm{Mo}, \mathrm{Mn}, \mathrm{Fe}, \mathrm{Zn}$ y B. El nitrato y el amonio se cuantificaron mediante electrodo selectivo, el $P$ por espectrofotometría de luz ultravioleta a $880 \mathrm{~nm}$ (Olsen \& Sommers, 1982), el Ca y Mg por espectrofotometría de absorción atómica y el $\mathrm{K}$ por espectrofotometría de emisión (USEPA, 1995). El Cu y el Mo se analizaron por ICP-Masas y el Mn, Fe, Zn y B mediante ICP-Óptico.

Además, con estos materiales se establecieron tres ensayos agronómicos de producción de planta ecológica de lechuga (Lactuca sativa L. var. Batavia Amarilla de París), perejil (Petroselinum crispum (Mill) Fuss) y albahaca (Ocimum basilicum L.). Se testaron los dos tratamientos iniciales (C y CG) conjuntamente con las mezclas que se elaboraron entre ambos al $75 / 25,50 / 50$ y $25 / 75 \%(v / v)$ (CG0 a CG100). Se sembraron dos bandejas de 40 alveolos de tamaño forestal por tratamiento en el mes de junio; la duración del cultivo fue de 3 meses. El cultivo se realizó bajo invernadero localizado en Lugo y el suministro de agua fue mediante riego por microaspersión y no se realizó fertilización adicional. Cuando se dio por finalizado el ensayo se cortaron las plantas para determinar el Peso fresco (PF) y Peso seco (PS) por alveolo tanto de la parte aérea como de la parte radicular aunque en las plantas de perejil la determinación de la biomasa de la parte radicular no fue posible llevarla a cabo debido al pequeño tamaño de las propias raíces y a la difícil separación del sustrato. El peso seco se determinó por secado en estufa a $60^{\circ} \mathrm{C}$ hasta peso constante. Además, a los dos meses de la puesta en marcha del ensayo, se midió el índice del contenido en clorofila mediante un medidor portátil CCM-200 (OptiSciences) que estima por absorbancia el contenido de clorofila en el tejido foliar. Sólo se tomaron datos en los cultivos de lechuga y albahaca, ya que dado el tamaño de la pinza no fue posible hacerlo en el cultivo de perejil.

Se empleó el MANOVA como tratamiento estadístico para los datos; siendo dos los factores principales o variables independientes: el cultivo y el porcentaje de compost con gallinaza a las mezclas. Se comprobó previamente la normalidad de los mismos mediante la prueba de Kolgomorov Smirnov y la homogeneidad de las varianzas mediante el contraste de Levene sobre la igualdad de varianzas. En aquellas variables donde el conjunto de datos no seguía una distribución normal, a éstas se les aplicó una transformación "Ln (x)". Como pruebas Post hoc se utilizó "Tukey", considerándose significativas las diferencias entre pares en los que la probabilidad del estadístico $F$ fue menor de 0,05. El programa informático utilizado para todo el tratamiento estadístico fue PASW Stadistics 18.

\section{Resultados y discusión}

\section{Caracterización de los compost}

La caracterización física de los materiales empleados a base de compost reveló una alta capacidad de aireación (CA) y drenaje frente a una baja disponibilidad de agua útil para los cultivos (Tabla 1). La CA, que supuso más de la mitad de la porosidad total $(90 \%)$, estuvo en unos valores 
cercanos al $50 \%$ y por tanto resultó bastante mayor de lo deseable. Por el contrario, la capacidad de retención de agua fácilmente asimilable (AFA) y de agua de reserva (AR) resultaron bajas. El parámetro $\mathrm{R}$ fue inferior a $1 \mathrm{kPa}$, lo que indica que el uso de estos compost como sustrato debería hacerse preferentemente en contenedores de pequeña altura (Ansorena, 1994). Teniendo en cuenta estas propiedades físicas, el uso exigirá un manejo del riego cuidado, con dosis pequeñas y frecuentes. La adición de gallinaza en el compostaje aumentó las densidades real y aparente, por el mayor contenido mineral de los compost resultantes; esto redundó en una porosidad algo menor, pero sin dejar de ser alta. Igualmente el compost con gallinaza (CG) presentó valores de CA y AFA significativamente mayores, y niveles de AR y ADA significativamente menores que el compost sólo de matorral (C).

\begin{tabular}{lrlrlc}
\hline & \multicolumn{1}{c}{ C } & \multicolumn{1}{c}{ CG } & & Óptimo \\
\hline $\mathrm{Dr}\left(\mathrm{g} \mathrm{cm}^{-3}\right)$ & $1,918 \pm 0,025$ & b & $2,078 \pm 0,019$ & a & $1,45-2,65$ \\
$\mathrm{Da}\left(\mathrm{g} \mathrm{cm}^{-3}\right)$ & $0,149 \pm 0,010$ & b & $0,218 \pm 0,016$ & a & $<0,4$ \\
$\mathrm{P}(\% \mathrm{v} / \mathrm{v})$ & $92,25 \pm 0,58$ & a & $89,50 \pm 0,79$ & b & $>85 \%$ \\
$\mathrm{CA}(\% \mathrm{v} / \mathrm{v})$ & $50,62 \pm 1,80$ & a & $54,23 \pm 7,43$ & a & $10-30$ \\
$\mathrm{AFA}(\% \mathrm{v} / \mathrm{v})$ & $7,63 \pm 1,07$ & b & $9,44 \pm 0,82$ & a & $20-30$ \\
$\mathrm{AR}(\% \mathrm{v} / \mathrm{v})$ & $2,85 \pm 0,18$ & a & $2,45 \pm 0,15$ & b & $4-10$ \\
$\mathrm{ADA}(\% \mathrm{v} / \mathrm{v})$ & $31,15 \pm 2,05$ & a & $23,38 \pm 6,70$ & b & \\
$\mathrm{R}(\mathrm{kPa})$ & $0,912 \pm 0,026$ & a & $0,838 \pm 0,110$ & a & $1-3$ \\
\hline
\end{tabular}

Tabla 1.- Principales propiedades físicas de los compost obtenidos ( $C$ = compost de matorral; $C G=$ compost de matorral y gallinaza). Valores medios \pm desviación típica y pruebas post hoc (Tukey).

1 Abad et al. (1993)
Solamente los tratamientos que contaron con un porcentaje de compost con gallinaza (\%CG) por encima del $50 \%$ mostraron unos valores de $\mathrm{pH}$ adecuados (en torno a 6,5) (Abad et al., 1993). La CE se mostró elevada en todos los compost, al igual que el valor de amonio, Ca y Mg (Tabla 2). El contenido en nitrato y $\mathrm{K}$ fue muy elevado. La concentración de $\mathrm{P}$ fue adecuada para los tratamientos con un porcentaje de compost con gallinaza en sus mezclas del $25 \%$ y $0 \%$; para los demás tratamientos este elemento se mostró un poco bajo; esto puede ser debido al incremento de $\mathrm{pH}$ registrado al aumentar el contenido de compost con gallinaza en las mezclas. Los niveles de $\mathrm{Cu}, \mathrm{Mn}, \mathrm{Zn}$, Mo y $B$ se encontraron dentro del rango óptimo recomendado por Abad et al., (1993), mientras que los niveles de Fe se localizaron ligeramente por debajo (datos no mostrados).

Como consecuencia de este alto contenido de elementos solubles el nivel de salinidad fue también elevado, mayor de lo deseable, subiendo de forma significativa en el que incorporaba gallinaza, donde se superaron los $7 \mathrm{dS} \mathrm{m}^{-1}$ en extracto de saturación.

Se cumplieron todos los requerimientos de la normativa para la obtención de la etiqueta ecológica comunitaria a sustratos de cultivo (Reglamento 2007/64/CE). Todos los compost estaban maduros y eran estables y quedaron completamente higienizados tras el compostaje (LópezLópez et al., 2009). En ningún caso se superó el contenido en elementos totales fijado por el reglamento y la CE medida en extracto 1:5 (v/v) estuvo por debajo del límite máximo de $1,5 \mathrm{dS} \cdot \mathrm{m}^{-1}$ (datos no mostrados).

\begin{tabular}{ccccccccc}
\hline Tratamiento & pH & $\mathrm{CE}$ & $\mathrm{NO}_{3}{ }^{{ }^{*}}$ & $\mathrm{NH}_{4}{ }^{+}$ & $\mathbf{P}$ & $\mathbf{K}$ & $\mathrm{Ca}$ & $\mathbf{M g}$ \\
\hline CG100 & 6,58 & 7,07 & $1.419,87$ & 67,76 & 4,15 & $1.265,50$ & 301,81 & 121,13 \\
CG75 & 5,99 & 6,43 & $1.337,13$ & 59,08 & 4,94 & $1.077,75$ & 294,73 & 121,70 \\
CG50 & 5,40 & 5,80 & $1,254,40$ & 50,40 & 5,74 & 890,00 & 287,66 & 122,28 \\
CG25 & 4,82 & 5,16 & $1.171,66$ & 41,72 & 6,53 & 702,25 & 280,58 & 122,86 \\
CG0 & 4,23 & 4,04 & $1,088,93$ & 33,04 & 7,33 & 514,50 & 273,50 & 123,44 \\
\hline Optimo & $5,2-6,3$ & $0,75-3,5$ & $100-200$ & $0-20$ & $6-10$ & $150-250$ & $>200$ & $>70$ \\
\hline
\end{tabular}

Tabla 2.- Valores medios de $\mathrm{pH}, \mathrm{CE}\left(\mathrm{dS} \mathrm{m}^{-1}\right)$, y elementos solubles $\mathrm{NO}_{3}^{-}, \mathrm{NH}_{4}{ }^{+}, \mathrm{P}, \mathrm{K}, \mathrm{Ca}$ y $\mathrm{Mg}(\mathrm{mg}$ $\mathrm{L}^{-1}$ ) en extracto de saturación de las mezclas de compost con gallinaza (CG) y sin gallinaza (C) en las distintas proporciones ensayadas, en comparación con el rango óptimo propuesto por Abad et al. (1993)

\section{Ensayos de cultivo}

Para el conjunto de los datos, existió un efecto significativo tanto para el factor \%CG en las mezclas como para el factor cultivo y la interacción entre ambos. Tanto en lo referido a los pesos frescos como secos y tanto para los tallos como para las raíces de las plantas $(\rho=0,000$ para todos los casos excepto para el peso seco radicular en donde la interacción "\%CG*cultivo" contó con un $\rho=0,030$ ).

El efecto del \%CG en el rendimiento de la biomasa fresca obtenida para el conjunto de los cultivos fue claro. Esto fue así en términos de crecimiento aéreo y también de crecimiento radicular (Tabla 3 ) ya que se observa que a medida que aumentó la proporción de estiércol en los sustratos también se incrementó significativamente el peso fresco de las plantas cultivadas al final del ensayo. Donde mejor se observa esta tendencia es en los valores de masa fresca de las partes aéreas de las plantas, que respondió de forma bastante lineal y continua. Sin embargo el incremento de tamaño de raíz de los planteles a medida que iba en aumento la proporción de gallinaza resultó ser un tanto más variable, evidenciándose solamente a partir de las mezclas CG75. Este efecto está en relación con el gran potencial fertilizante de la gallinaza; uno de los más potentes de entre todos los recursos naturales existentes (Kacar \& Katkat, 1999; Mengel \& Kirkby, 2001); basando su riqueza principalmente en nitrógeno (Simpson, 1990), fundamental para el crecimiento foliar de los cultivos en general (Arker \& Hills, 1980; Urbano, 1999).

Si se analizan separadamente los tres cultivos, se puede observar (Figuras 1, 2 y 3) como el orden en el crecimiento (en términos de peso fresco aéreo) fue el que cabía esperar según las características y el tiempo de cultivo: 
lechuga>albahaca>perejil. Los pesos frescos de los tallos de las plantas de lechuga estuvieron en el rango de 7-18 g, los de albahaca entre 2 y $7 \mathrm{~g}$, y por último los de perejil en el rango de 1 y $3,5 \mathrm{~g}$. En el cultivo de lechuga el crecimiento aéreo resultó superior en las mezclas con el $50 \%$ o más de compost con gallinaza respecto de los tratamientos con menor porcentaje de estiércol (Figura 1). La mezcla con mayor contenido en gallinaza (CG100) dio lugar a pesos secos más altos, mientras que en términos de peso fresco fue la mezcla CG50 la que alcanzó el máximo valor, debido a su menor contenido en materia seca. El peso seco radicular aumentó, aunque ligeramente, al incrementarse el contenido de compost con gallinaza en la mezcla, alcanzando los valores máximos en CG75 y CG100. Sin embargo en el peso seco esas diferencias resultaron inapreciables ya que los tratamientos con más gallinaza mostraron los menores contenidos de materia seca. El peso fresco de la parte aérea de las plantas de albahaca aumentó de CG0 a CG75, presentando este último tratamiento los pesos más altos junto con CG100 (estas dos mezclas resultaron ser estadísticamente iguales entre sí y superiores a las demás según el tratamiento post hoc empleado (Figura 2)). El peso seco aéreo siguió una tendencia similar, con máximos en CG75 y CG100. Por su parte el peso radicular presentó igualmente máximos en CG75, comparables a los obtenidos en CG100 y que contrastan con los mínimos obtenidos en CG25 y CG50; sin embargo el crecimiento en CG0 fue intermedio entre los dos grupos anteriores, rompiendo la tendencia.

\begin{tabular}{|c|c|c|c|c|c|c|}
\hline \multirow[b]{2}{*}{ CGO } & PF-P.Aérea & PS-P.Aérea & \multicolumn{2}{|c|}{ PF-P.Radicular } & \multicolumn{2}{|c|}{ PS-P.Radicular } \\
\hline & $3,74 \mathrm{c}$ & $0,46 \mathrm{~b}$ & 5,00 & $\mathrm{bc}$ & 0,67 & $a b$ \\
\hline CG25 & $4,20 \mathrm{~b}$ & $0,51 \mathrm{~b}$ & 3,42 & d & 0,55 & b \\
\hline CG50 & $5,80 \mathrm{~b}$ & $0,80 a$ & 3,97 & $\mathrm{~cd}$ & 0,66 & $a b$ \\
\hline CG75 & 7,53 a & $0,86 a$ & 7,73 & a & 0,89 & a \\
\hline CG100 & 7,02 a & $0,91 \mathrm{a}$ & 5,81 & $a b$ & 0,79 & a \\
\hline
\end{tabular}

Tabla 3.- Pesos frescos y secos $(\mathrm{g})$ de las partes aéreas y radiculares de las plantas; para el conjunto de los tres cultivos ensayados (lechuga, albahaca y perejil) atendiendo al porcentaje de compost con gallinaza en las mezclas. Valores medios y pruebas post hoc (Tukey)
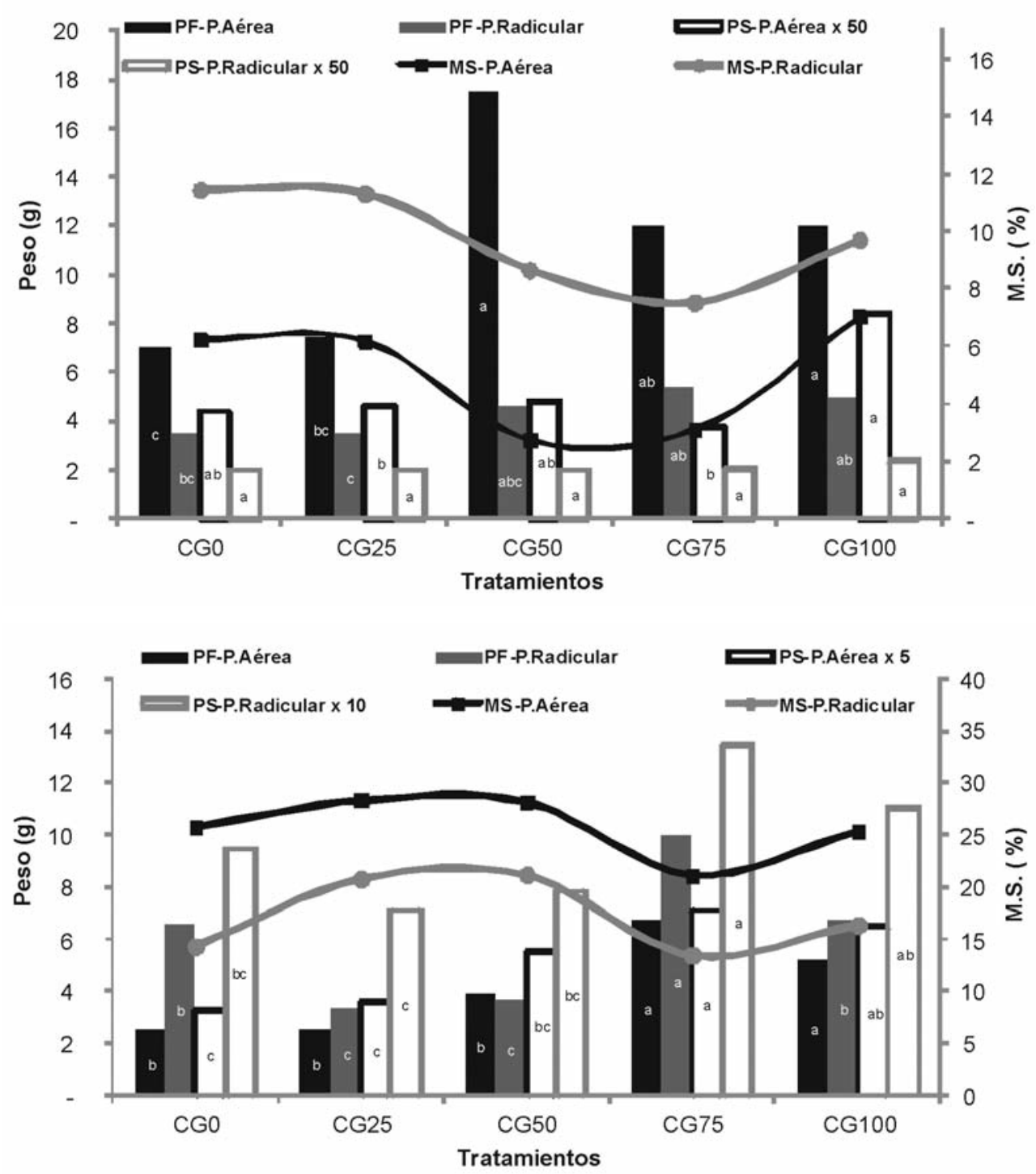

Figura 1.- Pesos frescos y secos de las partes aéreas y radiculares de las plantas de lechuga, y porcentaje de masa seca aérea y radicular, atendiendo al porcentaje de compost con gallinaza en las mezclas. Valores medios y pruebas post hoc (Tukey) para los pesos frescos y secos de la parte aérea y radicular; valores medios para los porcentajes de materia seca. Letras iguales para el mismo parámetro (columnas del mismo color) indican diferencias no significativas entre tratamientos para el nivel $p<0,05$

Figura 2.- Pesos frescos y secos de las partes aéreas y radiculares de las plantas de albahaca, y porcentaje de masa seca aérea y radicular, atendiendo al porcentaje de compost con gallinaza en las mezclas. Valores medios y pruebas post hoc (Tukey) para los pesos frescos y secos de la parte aérea y radicular; valores medios para los porcentajes de materia seca. Letras iguales para el mismo parámetro (columnas del mismo color) indican diferencias no significativas entre tratamientos para el nivel $p<0,05$ 
El incremento del porcentaje de compost con gallinaza en las mezclas dio lugar a un aumento significativo del peso seco aéreo de las plantas de perejil, que siguió una tendencia lineal (Figura 3). Por su parte el peso fresco presentó igualmente su máximo valor en CG100 y el mínimo en CG0, pero la tendencia en los tratamientos intermedios fue menos clara que en el peso seco, ya que hubo una oscilación importante en el contenido de materia seca para los distintos tratamientos.

Las diferencias entre las producciones en los sustratos con diferente contenido en estiércol para los distintos cultivos se puede deber a las diferentes exigencias nutricionales de los mismos, sobre todo a nivel de nitrógeno. Las dosis medias de nitrógeno extraídas a lo largo de un ciclo de cultivo, sitúan a las plantas de albahaca en las más exigentes en cuanto a éste término, seguidas de las de lechuga, y por último de las de perejil.

Economakis et al. (1997) y Martinetti et al. (1996) sitúan las necesidades de nitrógeno para un cultivo de lechuga en torno a los $100 \mathrm{~kg} \cdot \mathrm{ha}^{-1}$, dosis en la cual se produce la máxima producción de biomasa fresca; no viéndose ésta última incrementada con la aplicación de una mayor cantidad de nitrógeno (comprobándose con aportes de 150 a $200 \mathrm{~kg} \cdot \mathrm{ha}^{-1}$ ). Por lo que esto último puede explicar el porqué las plantas de lechuga pudieron no sufrir un incremento de peso sustancial al irse incrementando la cantidad de estiércol en las mezclas de sustratos donde fueron cultivadas a partir del tratamiento CG50. En cambio, para un cultivo de albahaca se recomiendan dosis de 150 $\mathrm{kg} \cdot \mathrm{ha}^{-1}$, aunque se siguen obteniendo valores más altos de biomasa hasta los $250 \mathrm{~kg} \cdot \mathrm{ha}^{-1}$ (Biesiada \& Kus., 2010), lo que puede explicar en este ensayo los incrementos de materia fresca hasta los \%CG mayores. Por otra parte, las necesidades de nitrógeno a lo largo de un cultivo de perejil son bastante bajas, situándose en el rango de 50-120 kg ha1 (Pasikowska et al., 2002); concretándose incluso en los $100 \mathrm{~kg} \cdot \mathrm{ha}^{-1}$ según los estudios de Krug (1991). Por lo que se puede explicar cómo ya a partir de la dosis más baja de gallinaza (CG25) se empiecen a obtener las mismas respuestas de crecimiento por mucho que ésta se incremente.

En cuanto al índice en contenido en clorofila medido para los cultivos de lechuga y albahaca (Figura 4), lo más importante a destacar es la diferencia significativa existente entre las medidas realizadas en las plantas cultivadas en sustratos que contaban con gallinaza (CG25, CG50, CG75 y CG100) frente a las plantas cultivadas en sustrato compuesto únicamente con tojo (CG0). Nuevamente se vuelve a relacionar este índice de contenido en clorofila con el estado nutritivo en $\mathrm{N}$ del cultivo (Madeira et al., 2003).

Como resultado de este trabajo se puede concluir que los sustratos elaborados a base de compost de tojo complementados en su elaboración con estiércol de gallinaza son aptos para el cultivo tanto de plantas hortícolas como aromáticas. De forma que en general, los mejores resultados se van obteniendo de manera creciente a medida que aumenta la dosis de estiércol en las mezclas. La pequeña cantidad de gallinaza $(5 \% \mathrm{v} / \mathrm{v})$ aportada al proceso de compostaje es suficiente para obtener unos buenos resultados de producción, ajustando el $\mathrm{pH}$ de los sustratos a unos valores adecuados, y sin poner en riesgo la obtención de la etiqueta ecológica comunitaria a sustratos de cultivo debido a un incremento de salinidad excesivo.

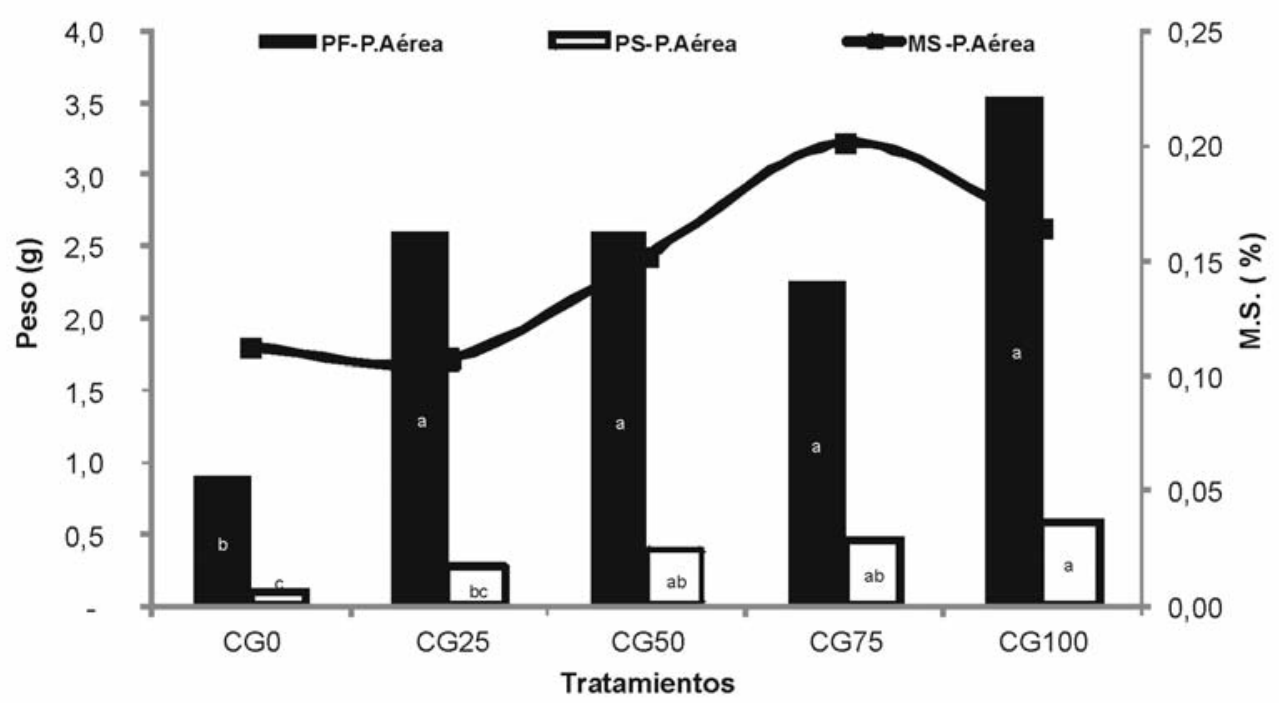

Figura 3.- Pesos frescos y secos de la parte aérea de las plantas de perejil, y porcentaje de masa seca aérea y radicular, atendiendo al porcentaje de compost con gallinaza en las mezclas. Valores medios y pruebas post hoc (Tukey) para los pesos frescos y secos de la parte aérea y valores medios para los porcentajes de materia seca. Letras iguales para el mismo parámetro (columnas del mismo color) indican diferencias no significativas entre tratamientos para el nivel $p<0,05$ 


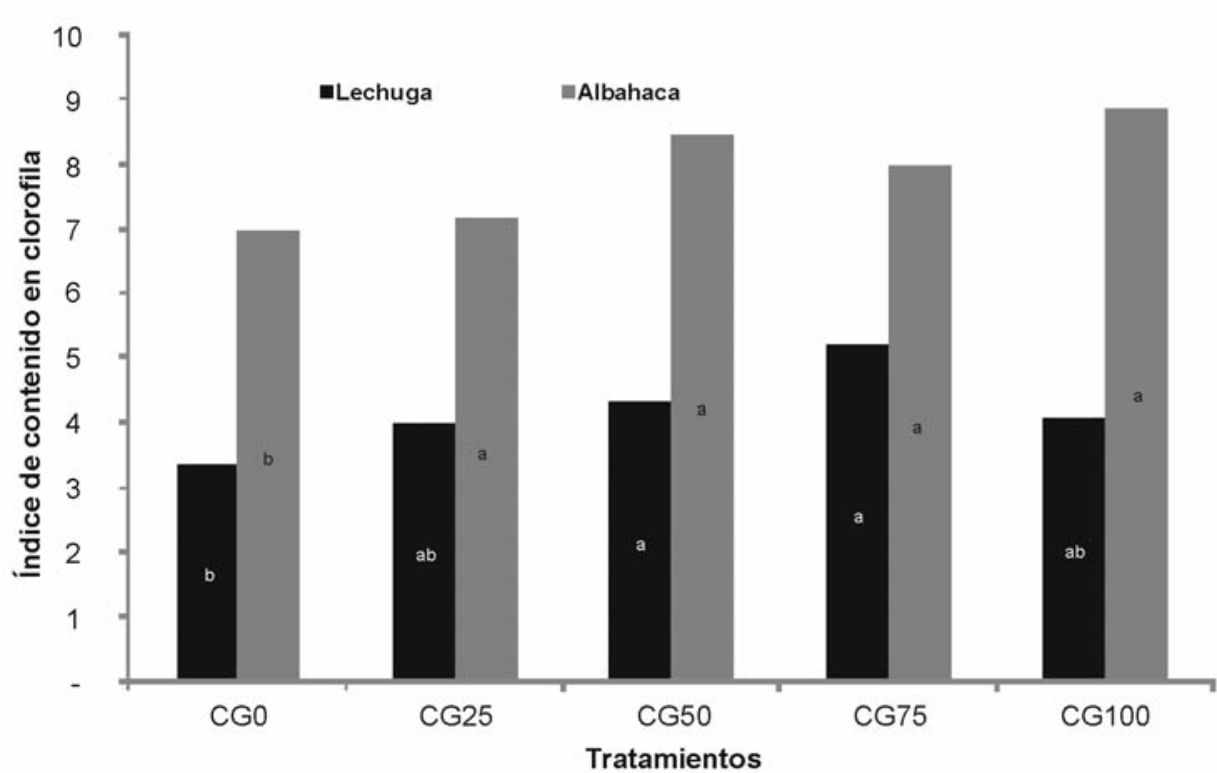

Figura 4.- Índice de contenido de clorofila de las plantas de lechuga y albahaca según tratamientos atendiendo al factor porcentaje de compost con gallinaza en las mezclas. Valores medios y pruebas post hoc (Tukey); letras iguales indican diferencias no significativas entre tratamientos para $p<0,05$
Agradecimientos Los autores agradecen a Transformacións Agrarias de Vilartó S.L. y a la Xunta de Galicia la financiación de este trabajo dentro del proyecto PGIDIT06RAG020E.

\section{Bibliografía}

Abad, M. (2002). Sustratos de cultivo en alvéolos, macetas y contenedores. p. 147-164. En: Ballester-Olmos J. J. F. (Ed.). Nuevas tecnologías en la viverística de plantas ornamentales. Generalitat Valenciana. Consellería de Agricultura, Pesca y Alimentación. Valencia.

Abad, M., Martínez, P.F., Martínez, M.D. \& Martínez, J. (1993). Evaluación agronómica de los sustratos de cultivo. Act.Hort. 11: 141-154.

Agulló, E., Bustamante, M.A., Paredes, C., Pérez-Murcia, M.D., Pérez-Espinosa, A. \& Moral, R. (2009). Utilización de compost vitivinícolas como sustituto total o parcial de la turba: propiedades de las mezclas y efecto en la producción en semillero. Actas de Horticultura 53: 96-101.

Ansorena, J. (1994). Sustratos. Propiedades y Caracterización. Ediciciones Mundi-Prensa. Madrid. 172 pp.

Arker, A. \& Hills, H. (1980). Ammoniun and nitrate nutrition of horticultural crops. Hort. Rev. Avi. 2: 395-423.

Biesiada, A. \& Kus, A. (2010). The effect of nitrogen fertilization and irrigation on yielding and nutritional status of sweet basil (Ocimum basilicum L.). Acta Sci. Pol., Hortorum Cultus 9 (2): 3-12.

Carmona, E. \& Abad, M. (2008). Aplicación del compost en viveros y semilleros. p. 397-424. En: Moreno J. \& Moral R. (Eds). Compostaje. Mundi-Prensa, Madrid.

Carmona, E., Moreno, M.T., Pajuelo, P., Martínez, M.D. \& Ordovás, J. (2009). Empleo del compost de orujo de vid como sustrato para semilleros de lechuga. Actas de Horticultura 53:121-125.
De Boodt, M., Verdonck, O. \& Cappaert, I. (1974). Method for measuring the water release curve of organic substrates. Acta Horticulturae 37: 2054-2062.

Economakis C.D., Koleilat R. \& Chartzonlakis K.S. (1997). Effect of nitrogen concentration on growth, water and nutrient uptake of lettuce plants in solution culture. Acta Horticulturae 449: 223-228.

Gómez, J., Minhorst, R. \& Piñeiro-Iglesias, I. (2009). Evaluación de diferentes sistemas de elaboración de sustratos de cultivo obtenidos a partir de tojo (Ulex euroapeus L.). Spanish Journal of Rural Development, 188204.

Iglesias, I., Rodil, C., Bessa, P. \& Lamosa, S. (2008). Gorse compost as a peat-substituve in growing media for the production of Thuja plicata "Zebrina". Acta Hort. 779: 615622.

Illera-Vives, M., López-Fabal, A. \& López-Mosquera, M.E. (2011). Evaluación de la fitotoxicidad de un sustrato a base de compost de algas y restos de pescado. Actas de Horticultura 59: 28-31.

Kacar, B. \& Katkat, V. (1999). Gübreler ve Gúbreleme Teknig i. VI'PAS, A.S, Bursa.

Krug H. (1991). Gemüseproduktion. Ein Lehr- und Nachschlagewerk für Studium und Praxis. Verlag Paul Parey. Berlin und Hamburg.

López-López, N.; López-Fabal, A. \& Amboaje-Fociños, J.L. (2009). Evaluación preliminar de mezclas de gallinaza y tojo para su uso como sustrato en agricultura ecológica. Actas de Horticultura 53: 135-139.

López-López, N.; López-Fabal, A.; López-Mosquera, M.E. \& Sainz-Osés, M.J. (2011). Composición de la solución de un sustrato a base de biomasa forestal compostada durante un cultivo de fresa en contenedor. Actas de Horticultura 59: 116-121. 
Madeira, A.C., Ferreira, A., de Varennes, A. \& Vieira, M.I. (2003). SPAD meter vs. Tristimulus colorimeter to estimate chlorophyll content and leaf colour in sweet pepper. J. Plant Nutrit. 34: 2461-2470.

Marchán, C.; Regueiro, M. \& Rubio, J. (2010). Panorama Minero 2008-2009. Instituto Geológico y Minero de España.

Martinetti L. (1996). Contenuto di nitrati e nitriti in lattuga (Lactuca sativa L.) al variare della concimacione azotata. Rivista di Agronomia 30: 92-96.

Mendoza-Hernández, D., García-de-la_Fuente, R., Belda, R.M., Fornes, F. \& Abad, M. (2011). Compostaje y vermicompostaje de residuos hortícolas: evolución de parámetros físicos y químicos durante el proceso. Consecuencias ambientales. Actas de Horticultura 59: 2227.

Mengel, K. \& Kirkby, E.A. (2001). Principles of Plant Nutrition. Kluwer Academic Publishers, The Netherlands.

Olsen, S.R. \& Sommers, L.E. (1982). Phosphorus. p. 403427. En: Page, A.L., Miller, R.H., Keeney, D.R. (Eds.). Methods of Soil Analysis. American Soc. of Agronomy, Madison, Wisconsin, USA

Ortega, M.C. \& Ordovás, J. (2011). Aprovechamiento de los lodos residuales de la industria del mármol como componente de sustratos de especies para fitorremediación. Actas de Horticultura 59: 38-41.

Pasikowska R., Browska B. \& Capecka E. (2002). The effect of nitrogen fertilization rate on the yield and quality of two cultivars of parsley (Petroselinum sativum L. ssp. crispum) grown on different soil types. Folia Hort. 14 (1): 177-185.
Simpson, T.W. (1990). Agronomic use of poultry industry waste. Poultry Science 70: 1126-1131.

Tapia, Y., Frutos, I., Eymar, E., \& Masaguer, A. (2009). Lixiviación de $\mathrm{Cd}$ y otros metales pesados en compost de restos de poda con biosólidos y compost de corteza de pino. Actas de Horticultura 53: 126-134.

UNE-EN-13041. (1999). Determinación de las propiedades físicas. Densidad aparente seca, volumen de aire, volumen de agua, valor de contracción y porosidad total. Mejoradores del suelo y sustratos de cultivo. AENOR.

UNE-EN-13652. (2002). Extracción de nutrientes y elementos solubles en agua. Mejoradores del suelo y sustratos de cultivo. AENOR.

Urbano, P. (1999). Tratado de fitotecnia general. Ediciones Mundi-Prensa. Madrid.

Urrestarazu, M., Mazuela, P.C. \& Alarcón, A.L. (2006). Cultivo en sustratos alternativos. p. 147-173. En: Alarcón A.L. (Coord.). Cultivos sin suelo. Compendios de Horticultura, Vol 17. Ediciones de Horticultura S.L., Reus.

USEPA. (1995). Test methods for evaluating solid wastes. Method 3051: Microwave assisted acid digestion of sediments, sludges, soils, and oils. Third edition. U.S. Environmental Protection Agency, Washington DC. 